\title{
An Analysis of Correlation Between Asymmetry of a Wave and Cognitive Behavior in Depression
}

\section{Ma Xiaomeng, Zhong Ning, Guo Jialiang, Zhang Minghui, Feng Lei, Fu Bingbing, Wang Gang, Zhou Haiyan}

Beijing University of Technology, International WIC Institute, Beijing, China

Email address:

zhouhaiyan@bjut.edu.cn (Zhou Haiyan)

${ }^{*}$ Corresponding author

\section{To cite this article:}

Ma Xiaomeng, Zhong Ning, Guo Jialiang, Zhang Minghui, Feng Lei, Fu Bingbing, Wang Gang, Zhou Haiyan. An Analysis of Correlation Between Asymmetry of $\alpha$ Wave and Cognitive Behavior in Depression. Science Innovation. Vol. 5, No. 4, 2017, pp. 231-237. doi: $10.11648 /$ j.si.20170504.17

Received: February 21, 2017; Accepted: May 3, 2017; Published: May 11, 2017

\begin{abstract}
Resting state brain waves of depression showed left-right asymmetry, which may be related to the strengthening of the right side of the brain. A typical cognitive disorder of depression is negative bias, especially outside information related to self, the phenomenon is more obvious. Recent studies have indicated that brain activity in resting state may reflect the intrinsic and essential function of brain function, and it is the foundation of cognitive activity. However, there was few study shew that left-right asymmetry about depression in resting state and whether the alpha wave is the foundation of negative bias. To Explore the relationship between asymmetry of $\alpha$ wave and cognitive behavior, we collected the EEG data behavior data in depressed people and healthy people. And analyzed the correlation between EEG data and behavior data in both depressed group and healthy group. The Result th was that a strong correlation between the self cognitive effects of the patient group and the self cognitive effects of the positive emotion and the left and right asymmetry of the resting EEG data. While the correlation between the self cognitive effects of control group were not related. These results further verify the relationship between the brain activity in resting state and cognitive activity, and it also reflects the relationship between the asymmetry of the alpha wave and the neural activity in the process of depression.
\end{abstract}

Keywords: Asymmetry, Negative Bias, EEG, Depression

\section{抑有症脑电 $\boldsymbol{\alpha}$ 波左右不对称性与认知行为的相关性研究}

马小萌, 钟宁, 郭家梁, 张明辉, 丰雷, 付冰冰, 王刚, 周海燕"

北京工业大学WIC研究院, 北京, 中国

邮箱

zhouhaiyan@b jut. edu. cn（周海燕）

摘要: 抑郁症的静息态脑电 $\alpha$ 波表现出左右不对称性, 这可能与大脑右侧负性活动加强有关。而抑郁症的一种典型认 知障碍是负性加工偏向, 尤其是外界信息与自我相关时, 该现象更加明显。近年来的研究表明静息态脑活动可能反映 了脑功能的内在的、本质的功能组织结构, 是认知活动的基础。然而关于抑郁症静息态 $\alpha$ 波的左右不对称性与是否是负 性自我加工偏向的基础关系还缺乏相关研究。本文为了探究抑郁症脑电 a 波左右不对称性与认知行为之间的关系, 采 集了抑郁症患者与健康对照组的静息态脑电数据和行为数据, 并在两组被试中均分析静息态脑电数据和行为数据之间 的相关性。结果表明患者组在自我条件下的情绪认知效应、和在正性情绪下的自我认知效应与静息态脑电数据 $\alpha$ 波的左 右不对称性都有很强的相关性; 而对照组的行为数据与静息态脑电数据 $\alpha$ 波的左右不对称性则没有相关性。这一结果进 
一步验证了静息态脑活动与认知活动的关系, 也反映出 $\alpha$ 波的左右不对称性可能是与抑郁症负性自我加工偏向的神经活 动基础直接关联性。

关键词： $\alpha$ 波左右不对称, 负性自我加工偏向, 脑电, 抑有症

\section{1. 引言}

近年来的认知神经科学研究表明静息态脑活动反映 脑内在的、具有本质性的功能组织活动, 可能是认知活动 的基础。从脑电的角度来看, 脑电的静息态脑活动通常记 录的是在闭眼休息的情况下, 从头皮采集的自发电信号。 抑郁症在静息态脑电中的一个典型表现是 $\alpha$ 波的左右不 对称性, 即与健康对照组相比, 抑郁症患者的 a 波的左侧 功率大于右侧 [2, 3]。研究表明, $\mathrm{a}$ 波功率与大脑的活动 强度负相关 [4], 即 $\mathrm{a}$ 波的功率越大, 大脑的活动越弱; $\alpha$ 波功率越小, 大脑的活动越强。由于 $\alpha$ 波的左右不对称 性是指左侧功率要大于右侧, 所以大脑左半球的活动要弱 于右侧, 即右侧活动强于左侧。这一特征实际反映了相对 于健康人, 抑郁症患者的大脑右侧活动强于健康人。有研 究表明, 大脑左侧活动可能与正性情绪加工有关, 右侧活 动与可能负性情绪加工有关 $[6,7]$ 。抑郁症的这种左右不 对称性表明了相对于健康人, 抑郁症患者由于大脑右侧活 动的加强, 其负性情绪的加工要强于健康人。并且, 由于 抑有症患者大脑右侧活动强于左侧活动, 说明抑郁症患者 的负性情绪加工可能强于正性情绪的加工。

从认知行为的角度来看, 抑郁个体往往对信息加工存 在负性偏向 $[6,7]$, 这可能是由于抑郁症的认知基础由否 定性的负性图式所构成, 因此比正常人更加关注负性信息, 故而在信息加工过程中会存在更多的偏差, 主要表现为注 意、记忆和解释偏向 $[11,12]$ 。，注意偏向是从选择性注 意的角度来考察抑郁个体认知偏向, 研究发现负性刺激被 抑郁患者优先加工并放大, 而其他刺激则容易被忽略, 这 种对负性刺激的注意偏向使抑郁症状得以持续和发展 (Baert, De Raedt, Schacht, \& Koster, 2010; Koster, De Raedt, Goeleven, Franck, \& Crombez, 2005; Gotlib, Krasnoperova, Yue, \& Joormann, 2004)。例如, 在信息 加工过程中表现出积极词汇认可量 (即被试看到标准词库 定义的积极词汇时, 是否自己本身将该词汇定义为一积极 词汇）低、加工速度慢 $[9,10]$; 回忆消极词汇效果（回忆 起消极词汇的数量) 好的特征 (Black \& Pösse1，2013; Connolly, Abramson, \& Alloy, 2015; Dozois, 2007; Goldstein, Hayden, \& Klein, 2014; Lemogne, 2009)。 记忆偏向一般指在记忆过程中, 抑郁个体在自由回忆任务 中更容易且更加偏向于回忆更多的消极词汇或事件 [13]。 解释偏向则指抑郁个体在解释模糊的刺激、情景和事件时, 往往以消极或者威胁性的方式来解释或理解 [15]。

而研究发现, 当外界信息与自我关联时, 这种认知偏 向更加明显。例如, 对自我相关的消极词的回忆速度快, 对积极词的加工速度慢的特征, 并且存在相对负性的自我 描述。也表明抑郁个体的自我图式中储存的积极信息较少, 消极信息较多 (Beck, 1976; Kelly \& Steven, 1990;
Morgan, 2010）。Beck（1976）认为，一旦外界刺激与自 我图式或知识结构一致，对此类信息的加工就更容易。个 体对外界刺激做出反应的过程中, 图式起着重要的作用。 个体对信息所做出的积极的抑或消极的解释不是偶然发 生的，而是一种与自身已存在图式有关的 “自传式” 的反 应 (Morgan, 2010)。这也揭示了抑郁个体对自我关联信息 的加工具有负性偏向是有其认知根源的, 即个体在头脑中 形成了负性自我图式。正是由于抑郁症个体形成了消极的 自我图式, 导致其对适合自我特征的刺激具有高度的敏感 性, 对这些刺激的加工快而且自信程度高, 并以消极的方 式编码个体经验, 倾向于对自我消极经验进行归纳, 而回 避情境中的积极因素, 进而对知觉产生歪曲、对信息产生 认知偏差(Williams, Watts, MacLeod, \& Mathews, 2001; 彭晓哲，周晓林，2005）。

尽管已有研究表明 a 波左右不对称性与大脑右侧负 性活动增强有关, 而抑郁个体又在认知上存在负性偏向性, 两者具有一定的关联性, 但是目前仍缺乏直接的证据来揭 示这种静息态脑活动于认知加工偏向之间的关系。因此, 在本研究中, 我们选择患者组与对照组两组被试, 分别采 集他们的静息态脑电数据和认知行为数据, 然后通过组间 比较考察 a 波左右不对称性与认知行为的关系。

\section{2. 对象和方法}

\section{1. 研究对象}

患者组来自正在安定医院住院的抑郁症患者。入组标 准: (1)右利手; (2)年龄18 60岁; (3)2周内未服用任何影响 神经系统功能的药物, 无酒精依赖或其他药物依赖; (4)由 2名精神科主治医师评估为抑郁症, 汉密尔顿抑郁量表 (Hamilton Depression Scale, HAMD) 评分>10; (5)无其 它精神疾病 (如精神分裂症、双向心境障碍等) 及严重躯体 疾病; (6)无严重的自杀倾向。排除标准: (1)有重大躯体疾 病、颅脑外伤或其他神经精神科疾病史者; (2)有严重焦虑 症状和惊恐发作的患者; (3)有自杀观念或自杀行为者。纳 入患者16例, 其中男 8 例, 女8例, 年龄18 58岁, 平均年 龄 $(43.44 \pm 13.27)$ 岁; 受教育年限 6 16 年, 平均 (12.13 13.36$)$ 年。

对照组来自周边的社区居民。入组标准: (1)右利手; (2)年龄18 60岁; (3)无抑郁症或其他精神疾病史; (4)2周内 未服用任何影响神经系统功能的药物, 无酒精依赖或其他 药物依赖。共招募16名对照, 其中男8名, 女8名, 年龄21 61 岁, 平均 (43.19 13.03) 岁; 受教育年限6 16年, 平均 (11.81 2 2.71) 年。 
患者组与对照组性别分布、年龄 $(t=0.95, P>0.05)$ 、 受教育年限 $(t=0.76, P>0.05)$ 的差异均无统计学意义。 本研究方案通过首都医科大学附属北京安定医院伦理委 员会审查, 所有被试自愿参加并签署知情同意书。抑郁症 病人数据收集于 2014 年 12 月, 收集地点为首都医科大学附 属北京安定医院。

\section{2. 研究方法}

\section{2. 1. 静息态EEG数据的获取}

实验过程中, 被试戴着电极帽坐在实验专用椅上, 保 持室内光线较弱, 环境安静, 被试处于闭眼静息状态, 记 录时间为4分钟。采集设备为Brain Products公司生产的 64 电极系统脑电采集系统, 所有电极的电阻降至 $5 \mathrm{~K} \Omega$ 以下, 采样频率为 $500 \mathrm{~Hz}$, 使用 $\mathrm{FCz}$ 作为参考电极 ( $\mathrm{REF}), \mathrm{AFz}$ 作为接地电极 (GND)。

\subsection{2. 行为数据的获取}

获取行为数据的任务为: 电脑屏幕上首先出现 “开 始” 字幕, 提醒被试注意力集中在电脑屏幕上; 随后出 现 “+” 号, 提醒被试刺激即将出现, 随后出现人称代词 “我” 或者 “他”; “+”号随即再次出现, 之后电脑屏 幕上出现一个情绪词, 这个词可能为正性, 也可能为负 性, 如 “高兴” 或者 “悲伤”; 随后 “+”号出现, 之后 电脑屏幕上出现情绪词的第一个字或第二个字，如 “高 兴” 的第一个字 “高” 或第二个字 “兴”，要求被试判 断该字在情绪词中的位置。如果为第一个字, 被试在反 应盒上按第一个键, 如果为第二个字, 被试在反应盒上 按第二个键。单字的出现到被试按键的时间即为反应时。 情绪词有两种词性, 正性（如：高兴）、负性（如：悲 伤），均选自现代汉语双子形容词感情信息评定表。在 本次研究中, 正性、负性词语各 48 个, 共 96 个词语。将 这96个词语平均分配到 4 组中, 每组包括 24 个词语, 其中 12 个正性词语, 12 个负性词语。

\section{3. 数据分析}

\subsection{1. 静息态EEG数据的分析}

EEG数据分析使用的是Brain Vision Analyser2. 0 。 具体包括: (1) 设置新的参考电极, 在这里我们使用TP9 和TP10两个通道作为新的参考电极。（2）去除眼电, 即 将被试眼动或眨眼带来的噪声人工手动去掉; (2) 去除 伪迹, 利用独立分量分析 (ICA) 将再实验过程中由于实 验设备或被试原因带来的干扰信号去除; （3）滤波，即 根据所要分析的信号频率, 适当设定波形带宽, 滤除不必
要的信号, 利用贷通滤波器进行 $0.5 \sim 30 \mathrm{~Hz}$; （4）根据汉 宁窗口进行分段, 将数据分为 $2.048 \mathrm{~s} /$ 段 (1.536s 重叠) 的数据段, 剔除幅值变化大于 $75 \mathrm{uV}$ 的数据段（5）快速傅 里叶变换 (FFT), 这样就将EEG由时域转换到频域, 本文中 使用得 $\alpha$ 波频段为 $8-13 \mathrm{~Hz}$; (6) 平均, 即将FFT后的数据 按汉宁时间窗口求平均。左右不对称分数公式为: $\operatorname{Ln}(\mathrm{R})-\mathrm{Ln}(\mathrm{L})$, 其中, $\mathrm{R}$ 为右侧电极点的功率, $\operatorname{Ln}$ 即对电极 点的功率取对数。本次试验用的是 64 电极帽, 去除两个参 考电极, 两个眼电, 还有其他数据不好的值, 最终每人得 到26个值, 即 26 组 a 波左右不对称性的值。

\subsection{2. 行为数据的分析}

衡量行为数据的指标为反应时, 即从反应刺激出现到 被试按键的时间。利用Eprime2.0采集被试的反应时，然 后将反应时导入 exce1以便处理。本研究共有四组实验条 件: 我正（代词为 “我”，情绪词为正性情绪词时）、我 负 (代词为 “我” , 情绪词为负性情绪词时) 、他正 (代 词为“他”, 情绪词为正性情绪词时)、他负 (代词为“他”, 情绪词为负性情绪词时），每组包括24个项目。我们首先 根据实验条件计算每个被试每组条件下平均反应时和标 准差, 并剔除极端值。极端值的标准为: 平均值 \pm 3 倍的 标准差, 即当数据小于平均值 -3 倍标准差或大于平均值 +3 倍标准差时, 即被定义为极端值, 然后剔除。然后根据抑 郁症组和健康对照组进行组分析。根据实验的条件, 分析 了几种实验的效应：（1）单个条件自身的实验效应（我 正，我负，他正，他负）;（2）自我条件下的情绪效应 （我正-我负）和他人条件下的情绪效应（他正一他负）; （3）正性情绪下的自我效应（我正一他正）和负性情绪下 的自我效应（我负一他负）。

\subsection{3. 统计学方法}

使用SPSS 20.0进行统计分析。患者组和对照组分别 做组内EEG数据左右不对称性与行为数据的Person相关性 分析, 得到相关性 $r$ 值, 并计算显著性 $P$ 值: 当 $P>0.05$ 时, 说明EEG数据左右不对称性与行为数据的相关性不显著, 即无统计学意义; 当 $P<0.05$ 时, 说明 $E E G$ 数据左右不对称 性与行为数据的相关性显著, 即有统计学意义。

\section{3. 结果}

分析结果表明患者组 $\alpha$ 波左右不对称性与认知行为 具有相关性, 而对照组两者之间不具有这种相关性, 具体 结果如下。 


\section{1. 患者组 $\boldsymbol{\alpha}$ 波左右不对称性与认知行为的相关性分析}

3. 1. 1. 自我条件下情绪认知效应与 a 波左右不对称性的相关

(a)

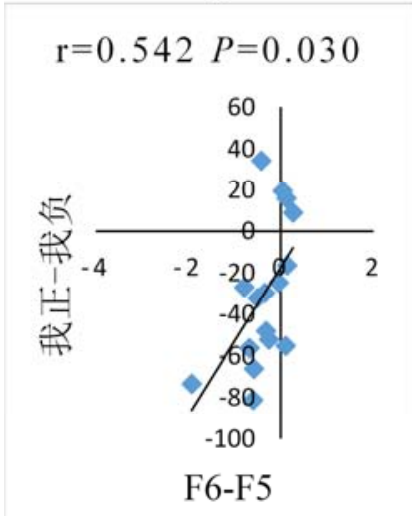

(b)

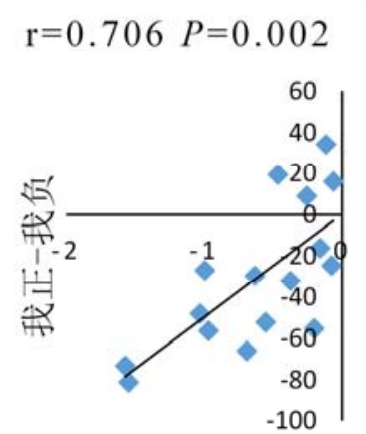

FC6-FC5 (c)

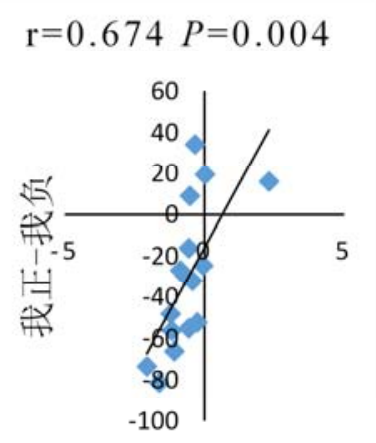

FT8-FT7

图1 患者组自我条件下的情绪效应（我正-我负）与 a 波左右不对称性的相关性。其中：（a）表示我正-我负与F6-F5的相关性；（b）表示我正-我 负与FC6-FC5的相关性; (c) 表示我正-我负与FT8-FT7的相关性。F6-F5, FC6-FC5与FT8-FT7均表示EEG数据 $\operatorname{Ln}(\mathrm{R})-\operatorname{Ln}(\mathrm{L})$ 之后得到的数值。

我正-我负表示在自我条件下不同情绪词的加工效应。如图1所示: 在自我条件下，情绪词与F6-F5的相关存在统计 学意义 $(\mathrm{r}=0.54, P<0.05)$; 与FC6-FC5的相关存在统计学意义 $(\mathrm{r}=0.71, P<0.05) ;$ 与FT8-FT7的相关存在统计学意 义 $(\mathrm{r}=0.67, P<0.05)$

3.1.2. 正性情绪条件下自我效应与 a 波左右不对称性的相关

(a)

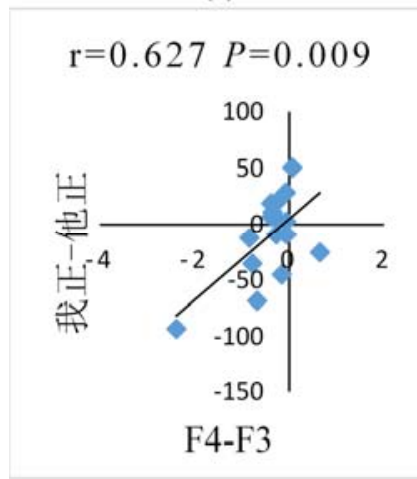

(d)

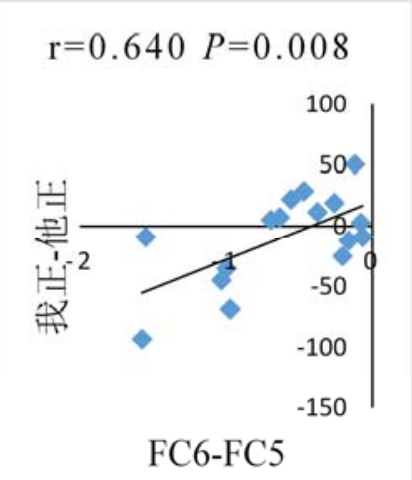

(b)

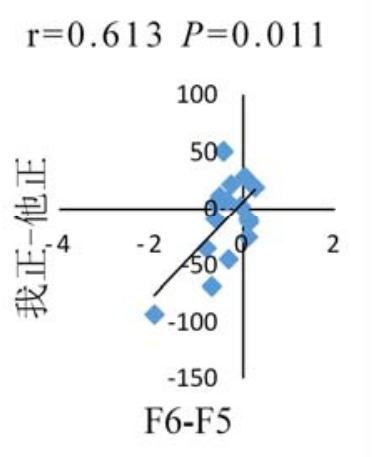

(e)

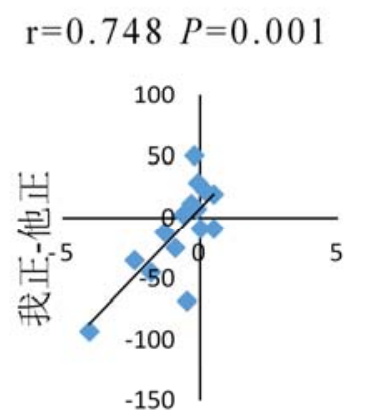

TP8-TP7 (c)

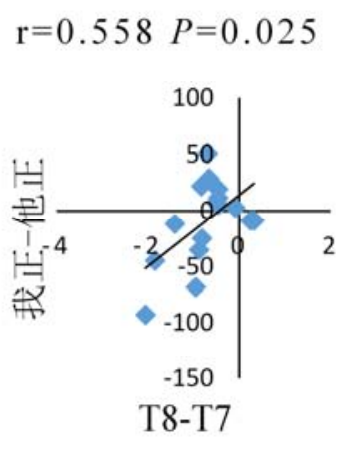

图2 患者组正性情绪条件下自我效应（我正-他正）与 a 波左右不对称性的相关性，其中：（a）表示我正-他正与F4-F3的相关性；（b）表示我正他正与F6-F5的相关性; （c）表示我正-他正与T8-T7的相关性;（d）表示我正-他正与FC6-FC5的相关性; (e) 表示我正-他正与TP8-TP7的相关性。 F4-F3, F6-F5, T8-T7, FC6-FC5, TP8-TP7均表示EEG数据Ln (R)-Ln (L) 之后得到的数值。 
我正-他正表示在正性情绪词下自我的影响。由图2 得到结果: 在自我条件下, 自我与F4-F3的相关存在统计 学意义 $(r=0.63, P<0.05)$; 自我与 $F 6-F 5$ 的相关存在统 计学意义 $(r=0.61, P<0.05)$; 自我与 $T 8-T 7$ 的相关存在 统计学意义 $(r=0.56, P<0.05)$; 与 $F C 6-F C 5$ 的相关存在 统计学意义 $(r=0.64, P<0.05)$; 与TP8-TP7的相关存在 统计学意义 $(r=0.75, P<0.05)$

\section{2. 对照组EEG数据左右不对称性与行为数据的相关性分析}

对照组健康被试的EEG左右不对称性与自我参照的相 关性均不存在统计学意义 $P>0.05$.

\section{4. 结论}

近年来, 精神疾病患者静息态脑功能的研究渐渐引起了 重视。静息态脑活动作为基础状态下的大脑活动, 有较好的 研究及临床利用价值。而且, 其作为大脑活动的基础, 可能 与认知行为有关。抑有症患者静息态脑功能FMRI研究发现, 在静息态下, 大脑前额背侧与认知执行功能有关。该研究亦 发现, 该区域有潜力用于预测抑郁症患者的阻滞、认知损伤 症状, 随病期与发作次数而变化。另外亦有研究发现抑有症 患者眶额皮质活动的增强可能与患者在静息态时仍存在的 强烈的情绪加工、自我意识、内省等活动有关, 抑郁症患者 可能会动用强烈的情绪加工、控制来维持其认知表现。颢叶 与感知信息的加工有关。这些感知信息内容广泛, 包括视觉、 听觉相关的言语理解、识记, 音乐加工、面部感知等。这些 脑区功能的正常运行是执行社会功能正常的保证。这些都说 明静息态脑活动与认知行为存在很大的相关性联系。

本研究主要通过采集抑郁症组与正常组的静息态脑 电数据与行为数据, 考察静息态脑电活动于认知行为的关 系。结果表明: 相较于健康人, 抑郁症患者的 a 波左右不 对称性与认知行为具有更强的相关性, 这些结果进一步验 证了静息态脑活动与认知活动的关系, $\alpha$ 波的左右不对称 性可能是抑郁症负性自我加工偏向的神经活动基础。抑郁 症患者大脑右侧负性活动的加强影响了其对外界信息的 认知加工, 从而产生异于常人的认知活动, 为我们研究抑 郁症提供了可靠地依据和方向。

Davidson的规避-接近系统模型认为, 右侧活动属于 规避行为系统, 与负性情绪加工有关, 左侧活动属于接近 行为系统, 与正性情绪加工有关 $[22,23]$ 。因此, 左右不 对称性是与大脑右侧负性活动增强有关。左右不对称性反 映抑有症的接近行为动机系统受损的特征 [6], 这一推论 与抑有症快感缺失和兴趣减退的症状相一致 [38]. 即抑郁 症患者的左侧活动小于右侧活动的原因是其有意的回避 整形情绪的加工, 有意偏向于负性情绪的加工 $[25,26]$, 刚好与抑郁症患者的负性偏向性相一致。

\section{5. 讨论}

关于抑郁的负性认知偏向, 不同的研究存在不同的观 点。Beck (1987) 最先倡导抑郁认知理论, 他认为抑郁个体
的认识活动或认识过程是消极的, 能恰当的用来解释抑有 个体自我参照加工过程所表现出的消极认知偏向 [28]。他 无其强调三类导致抑郁的消极认知, 即抑郁认知三联征: 个体总是对自我做消极评价、对以往经验做消极解释、对 未来做消极预期。这些认知特征与临床抑郁个体在自我参 照加工过程中的表现极相符。并且它与 “认知歪曲”、“自 动性思维” 和 “潜在的抑郁性认知图式作为核心概念来解 释抑郁认知偏向的产生。认知歪曲是指个体对客观现实的 错误解释或错误知觉。在这种情况下, 抑郁个体得出的结 论会使他们的消极期望得到进一步加强。Bower（1981） 的情绪记忆的语义网络模型认为, 每一种基本情绪 (如愉 快、愤怒、恐惧等) 在记忆网络中是以结点的形式表征的, 复合情绪由基本情绪的许多结点同时激活构成。每一个基 本情绪的结点都与其他许多结点相联系, 如表情行为、自 主反应模式、评价唤醒、语言标签等。在情绪的语义网络 中, 每种情绪被表征为一个相互联系的记忆网络中的一个 节点。一个节点的激活会增加相联系节点的通达, 从而产 生一个加工偏向, 偏向与加工与情绪状态相一致的信息 $[28,29]$ 。例如在Bower 等1978 年的一项研究中, 诱导愉 快和悲伤两种心境, 然后要求被试回忆童年时期的事情, 结果发现在愉快心境下的被试回忆出更多童年时期愉快 的事件, 而在悲伤心境下的被试回忆起了更多的童年时期 不愉快的事件 (Bower, Monteiro, \&Gilligan, 1978)。由 此可以理解, 抑郁患者因长期处于负性情绪状态, 其语义 网络节点易激活负性加工偏向, 进而产生认知偏向。比如 抑郁个体自传体记忆中与自我相关的负性生活事件更易 被激活而得到加工, 进而导致与其相联结的负性记忆网络 节点的通达, 抑郁情绪随之产生并持续发展 [27]。认知负 荷理论认为, 人们在进行信息加工时, 认知资源是一定的, 即在同一时间内只能有效地进行一项细淢活动, 而其他任 务则会被暂时搁置, 当负性刺激与其他刺激一起出现时, 注意资源极易被负性刺激所吸引、占用, 此时就不能很好 的完成其余任务, 从而表现出负性注意偏向 [36]。例如, Garner任务中, 抑郁患者在进行情绪面孔的性别判断任务 是, 收到面孔情绪信息的干扰, 抑郁个体消耗了注意资源, 因而不能很好地完成性别判断任务, 表现为性别命名时间 延长, 从而出现负性注意偏向。

为了进一步考察 $\mathrm{a}$ 波左右不对称性与认知行为的关 系, 还需要做更多的工作。例如, 本研究由两组被试组成, 每组被试的数量为 16 人, 虽然做到了两组被试在性别, 年 龄等方面的匹配, 仍需要进一步扩大样本来增加说服力; 其次, 静息态脑功能的 fMRI研究也发现, 在静息态下, 大 脑前额背侧与认知执行功能有关。在本实验中, 抑有症组 自我条件下的情绪效应与 3 对 $\alpha$ 波左右不对称性（F6-F5, FC6-FC5 与FT8-FT7) 具有很强的相关性, 看上去主要分布 在前部额叶地区, 而前部额叶是自我和执行控制的脑区, 与fMRI研究具有一致性。但由于脑电难以对脑活动进行精 细地空间定位, 需要进一步采用脑电空间定位的方法 (例 如sLoreta) 来定位具体与行为认知相关的脑区。所以在以 后的研究中, 我们会尽量提高我们的样本数量, 并且对具 体脑区做精确地定位。从而更好地给抑郁症的研究提供精 确而可靠地理论支持和方向。 


\section{致谢}

项目基金: 国家重点基础研究发展计划资助(编号: 2014CB744600); 国家国际科技合作专项资助（编号: 2013DFA32180）; 国家自然科学基金资助项目（编号: 61420106005, 61272345）; 北京市教育委员会资助; 磁共 振成像脑信息学北京市重点实验室资助; 北京市自然科学 基金面上项目 （编号：4132023）

\section{参考文献}

[1] KEMP A H, GORDON E, RUSH A J, et al. Improving the prediction of treatment response in depression: integration of clinical, cognitive, psychophysiological, neuroimaging, and genetic measures [J]. CNS Spectrums, 2008, 13 (12) : 1066-1086.

[2] Diego M A, Field T, Hernandez-Reif M. CES-D depression scores are correlated with frontal EEG alpha asymmetry. Depression Anxiety, 2001, 13: 32-37.

[3] Cook I A, 0' Hara R, Ui jtdehaage S H J, et al. Assessing the accuracy of topographic EEG mapping for determining local brain function. Electroencephalogr. Clin Neurophysiol, 1998, 107: 408-414.

[4] Mogg, K., Bradbury, K. E., \& Bradley, B. P. Interpretation of ambiguous information in clinical depression. Behav Res Ther, 2006, 44, 1411-1419.

[5] Mogg, A., Pluck, G., Eranti, S. V., Landau, S., Purvis, R., Brown, R. G., et al. A randomized controlled trial with 4-month follow-up of adjunctive repetitive transcranial magnetic stimulation of the left prefrontal cortex for depression. Psychol Med, 2008, 38, 323-333.

[6] Beck, A. T. Cognitive Therapy and the Emotional Disorders. New York: International Universities Press. 1976

[7] Beck, A. T. Cognitive Models of Depression. Journal of Cognitive Psychotherapy, 1987, 1, 5-37.

[8] Everaert, J., Koster, E. H., \& Derakshan, N. The Combined Cognitive Bias Hypothesis in Depression. Clinical Psychology Review, 2012, 32, 413-424.

[9] Gotlib, I. H., Krasnoperova, E., Yue, D. N. , \& Joormann, J. Attentional Biases for Negative Interpersonal Stimuli in Clinical Depression. Journal of Abnormal Psychology, 2004, 113, 127-135.

[10] Blaut, A., Paulewicz, B., Szastok, M., Prochwicz, K. , \& Koster, E. Are Attentional Bias and Memory Bias for Negative Words Causally Related? Journal of Behavior Therapy and Experimental Psychiatry, 2013 , 44, 293-299.
[11] Phillips, W. J., Hine, D. W., \& Thorsteinsson, E. B. Implicit Cognition and Depression: A Meta-Analysis. Clinical Psychology Review, 2010, 30, 691-709.

[12] Moser, J. S., Huppert, J. D. , Foa, E. B. , \& Simons, R. F. Interpretation of Ambiguous Social Scenarios in Social Phobia and Depression: Evidence from Event-Related Brain Potentials. Biological Psychology, 2012, 89, 387-397.

[13] Taylor, J. L., \& John, C. H. Attentional and memory bias in persecutory delusions and depression. Psychopathology, 2004, 37, 233-241.

[14] Laufs H, Kleinschmidt A, Beyerle A, et al. EEG-correlated fMRI of human alpha activity. Neuroimage, 2003, 19: 1463-1476

[15] Davidson R J. Affective neuroscience and psychophysiology: Toward a synthesis. Psychophysiology, 2003, 40: 655-665

[16] Symons C S, Johnson B T. The Self - reference Effect in Memory: A Meta - analysis [ J ] Psychology Bulletin, 1997, 121(3): 371-394.

[17] Berlad I, Pratt H. P300 in Response to the Subject' s Own Name [J] . Electroencephalography and Clinical Neurophysiology/Evoked Potentials Section, 1995, 96 (5) : 472.

[18] Schneider M. Comparing the Neural bases of Self referential Processing in Typically Developing and 22q11. 2 Adolescents [J] . Dev Cogn Neurosci, 2012, 2 (2) : $277-289$.

[19] ] Kim H. A Dual - subsystem Model of the Brain' s Default Network: Self - referential Processing, Memory Retrieval Processes, and Autobiographical Memory Retrieval [J]. Neuroimage, 2012, 61(4) : $966-977$.

[20] Trafimow D, Triandis H, Goto S. Some Tests of the Distinction Between the Private Self and the Collective self [J]. Journal of Personality and Social Psychology, 1991, 60( 5): 649-655.

[21] JA Coan, JJB Allen. Frontal EEG asymmetry and the behavioral activation and inhibition systems. Psychophysiology, 2003, $40(1): 106-14$.

[22] JJB Allen, HL Urry, SK Hitt, JA Coan. The stability of resting frontal electroencephalographic asymmetry in depression. Psychophysiology, 2004, 41(2):269280.

[23] JK Gollan, D Hoxha, et al. Frontal alpha EEG asymmetry before and after behavioral activation treatment for depression. Biological Psychology, 2014, 99(1): 198-208. 
[24] JA Coan, JJB Allen. Frontal EEG asymmetry as a moderator and mediator of emotion. Biological Psychology, 2004, $67(1-2)$ : 7-50.

[25] SW Black, P Pössel. Integrating Beck's Cognitive Model and the Response Style Theory in an Adolescent Sample. Journal of Youth \& Adolescence, 2014, 44(1) : $1-16$.

[26] GH Bower, KP Monteiro, SG Gilligan。 Emotional mood as a context for learning and recall。Journal of Verbal Learning \& Verbal Behavior, 1978, 17(5) : 573-585.

[27] 王建. 抑郁个体认知偏差的研究进展. 心理学进展, 2016, $6(2), 169-180$ 。

[28] 王晓霞, 蒋成刚, 李静, 等. 抑郁症患者对正性情绪图片 增强和减弱认知重评的fMRI研究. 中国临床心理学杂志, 2015, 23，615-575。

[29] 胡治国, 刘宏艳, 卓永宁, 等. 抑郁症患者的情绪冲突研 究. 中国临床心理学杂志. 2010, 18, 37-40。

[30］吴贝贝，曹召伦，何成森. 抑郁症的认知行为疗法研究现 状. 安徽医药 Anhui Medical and Pharmaceutical Journal 2011 Mar; 15 (3)。
[31] 廖成菊, 冯正直. 抑有症情绪加工与认知控制的脑机制. 心 理科学进展2010, Vol. 18, No. 2, $282-287$ 。

[32] 杨文静, 刘培朵, 崔茜, 等. 自我参照对情绪性记忆定向 遗忘的影响. 心理学报 2014 , Vol. 46, No. 2, 156-164。

[33] 廖智舟, 李川, 周军, 等. 抑郁症静息态EEG 前后部脑电 活动. 智能系统学报 CAAI Transactions on Intelligent Systems Apr. 2014。

[34] 刘雷, 周仁来. 一个测量抑郁症的重要神经指标：静息额 叶脑电活动的不对称性. 心理科学进展 2015, Vol.23, No. $6,1000-1008$ 。

[35] 朱熊兆, 钟明天, 蚁金瑶, 等. 临床抑郁症患者的注意偏 倚特征. 中国临床心理学杂志. 2008, 16, 234-236, 250。

[36] 王丽。抑郁症患者静息态的脑功能磁共振研究。

[37] 郭家梁, 付冰冰, 周海燕, 冯媛, 丰雷, 等。抑郁症静息 态脑电的前额不对称性分析。精神医学杂志, 2016 (2)。 